\title{
A Supervised Classification Techniques to Optimize Error Evaluation and Space Complexity
}

\author{
M.Santhiya, M.Shobana, R.Jegatha
}

\begin{abstract}
Bayesian classification is based on Baye's Theorem, which is applied on a conditional probability basis of posterior and prior probabilities in parallel with future evidence. Prior Probabilities are the original probabilities of an outcome which will be updated with new information to create posterior probability. The revised probability of an event occurring after taking into consideration new information.A Bayesian classifier is used to predict the values of features for members of that class. It is used to over come the diagnostic and predictive problems. This classification provides a useful perspective for understanding and evaluating machine learning algorithms.It is a probabilistic learning algorithm which calculates the explicit probabilities for hypothesis, among the most common learning problem.The proposed work has focused on designing of two classification algorithms naïve space and naïve Mine classification to optimize space complexity and error evaluation forlarger data sets.
\end{abstract}

IndexTerms- Prior \& Posterior Probability, Bayes Theorem, Naive Space, Naive Mine.

\section{INTRODUCTION}

The data or information that is anticipated in the present situation is significantly arranged or characterized. Arrangement goes under regulated learning methods of AI. Characterization can be quickly portrayed as the undertaking of doling out a class to occasions of information depicted by a lot of characteristics. It incorporates the development of a classifier which is prepared on a lot of preparing information that beforehand has the right class alloted to every datum point. Arrangement fabricates a brief model of the appropriation of class names and afterward used to group new information where the estimations of highlights are known however the class is obscure. Bayesian arrangement depends on Bayes hypothesis. Bayesian hypothesis gives a numerical math of conviction, which depicts what it implies for convictions to be reliable and how they should change with evidence.This supposition, called class restrictive freedom, which is made to improve calculation, thus it isconsidered 'Naive'.Bayesian classifiers are the statisti calclassifiers which predicts class participation probabilities. Guileless Bayes classifier works best in two cases, When the highlights are totally autonomous and also when the highlights are practically needy.

Revised Manuscript Received on August 05, 2019.

M.Santhiya, Assistant Professor, Rajalakshmi Engineering college, Chennai, Tamilnadu, India.

(Email: santhiyamunusamy@gmail.com)

M.Shobana, Assistant Professor, Saveetha Engineering college, Chennai, Tamilnadu, India.

(E-mail:mshobana2008@gmail.com).

R.Jegatha, Assistant Professor, Sri Sairam Institute of technology, West Thambaram, Chennai, Tamilnadu, India.

(E-mail: jegatha.it@sairamit.edu.in).

\section{LITERATURE SURVEY}

Bayesian characterization [1] with Mistake Adapted successive testing system. Earlier Learning can be utilized related to the preparation information to build the ideal Bayesian Classifier. Discovering more exactness in forecast of information ought to be improved. To present grouping and bunching systems and execution investigation with exactness in blunder detection.Spatial parallel order, Spatial summed up direct model (SGLM)[2] and the Bayesian spatial summed up straight blended model (SGLMM) is utilized to recoup information robustness. A Bayesian characterization for perceiving written by hand numerical articulations [3]. Presenting some constraintson how data sources might be parceled, [4]we inferred an effective parsing calculation got from Unger's method.Expert elicitation and Bayesian System demonstrating for transportation Mishaps. How BBN is connected for demonstrating dangers in the oceanic area, uncommonly on where information originate from and how they are connected. A Bayesian approach [5],

forcharacterizing vulnerability in announcing apopulation breakdown, this gauge of vulnerability as an approach to set a certainty interim around a predefined rate decay from the most extreme. Probabilistic methodology [6]for Anticipating the Size of Coding Units in the Quad-Tree Structure of the Quality and Spatial Versatile HEVC. An improved pressure execution atthe cost of critical increment in computational codingcomplexity. On Bayesian Network

Classifiers[7]with Decreased Exactness Parameters, Execution is better wavelet-based improved Bayesian surmising. Precision and execution in system model ought to be expanded for discriminatively improved parameters for everything except extremely low piece widths. Moving endlessly from Blunder Related possibilities [8] to accomplish spelling remedy in P300 spellers. For bigger informational collections mistake must be assessed and improve the precision. its utilization as a compelling specialized instrument is dependent on high P300 arrangement exactnesses $70 \%$ to represent mistake revisions. Generalized different bit learning [9] with Information Subordinate Priors. Earlier likelihood of blunder ought to be improved with exactness. Early interterm flaw diagnosis[10] in acceptance machines utilizing an explanatory.

Published By: 


\section{PROPOSED WORK}

A. Naive bayes space algorithm

The Naive Bayes space algorithm is to reduce space complexity.In Fig1, the selected Input file is preprocessed and undergoes space evaluation to minimize memory space.

\section{Pseudocode for Naive Space Classifier:}

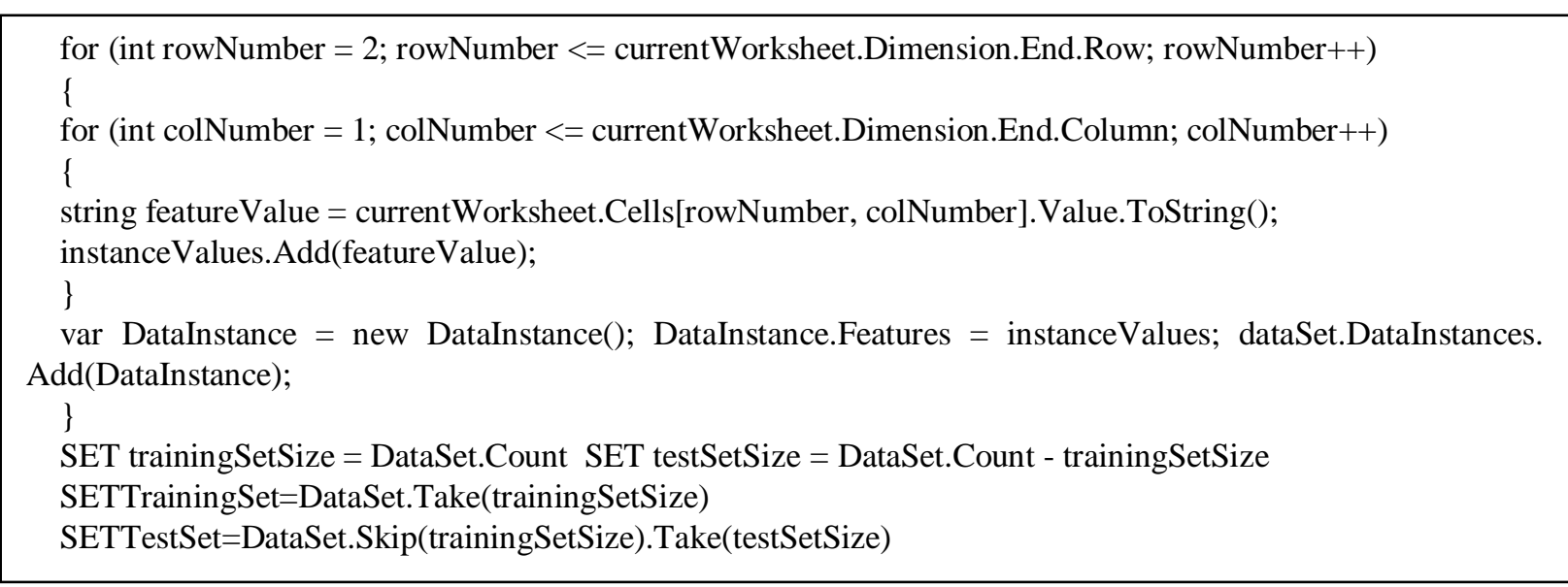

\section{B. Naive bayes mine algorithm}

The Naive Bayes Mine algorithm calculatesPrior Probability and Posterior Probability for each data sets.

Pseudocode for Naive Mine Classifier:

Compute PriorProbability as ClassCount / TotalCount

FOR EACH ClassProbability Pc in PriorProbabilityList

\{

SET posterior Probability $=\mathrm{Pc}$

FOREACH Conditional Probability CPi in ConditionalProbabilityList

\{

Compute posterior Probability as posterior Probability * CPi

Add posteriorProbability to PosteriorProbabilityList

\}

\section{C.Flow Analysis Of Algorithms}

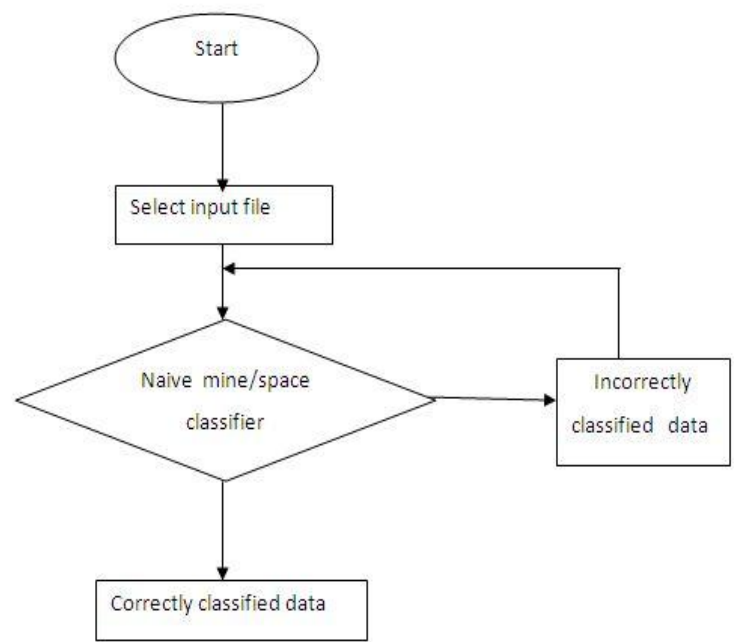

\section{ARCHITECTURE DIAGRAM}

A trained data sets of information is utilized to assess the relationship among different informational data set collections. In Fig2, Preparing sets are utilized in canny frameworks, AI, genetic programming and measurements. These preparation sets are then sent into the preprocessing unit to evacuate boisterous data.The data which is to be assessed contains dirty and fragmented garbage esteems, total information and trash esteems with mistakes and anomalies. Once preprocessing is finished with numerous means so as to improve the nature of the information and simplicity of procedure. Preprocessing includes Information cleaning, Information Coordination,

Fig1. Flow Chart of Naïve Space/Mine Algorithm 
Information Change, Information reduction. The Preprocessed information is presently changed over to ARFF (Attribute-Relation File Format) document which is an ASCII content document .The preprocessed record comprises of a rundown of cases with a lot of properties as .arff record group. The anomaly is removed and cleaned information is sent into the Bayes classifier and grouped as needs be.

Grouping is done dependent on space and mine classifier and various yields are gotten.

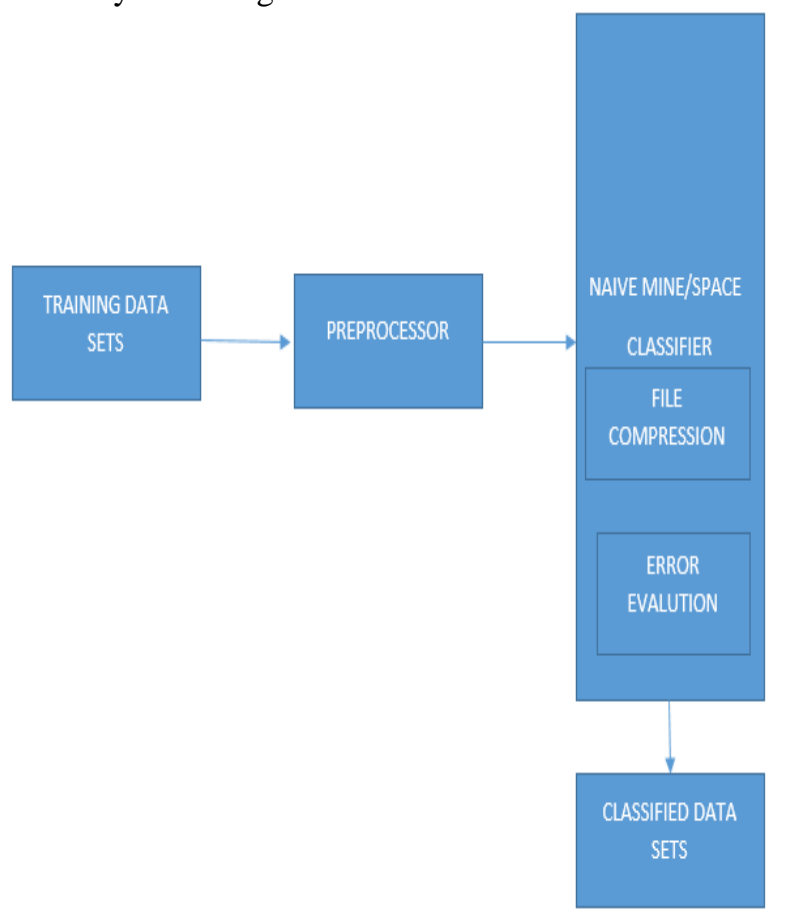

Fig. 2 Architecture diagram of the proposed system

\section{EXPERIMENTAL RESULTS}

The Proposed algorithm provides a feasible solution for calculating the accuracy rate for the attribute sets. The pictorial representation Fig. 3 of Naive algorithm yields $30 \%$ accuracy rate directly proportional added with increased accuracy rate.Accuracy analysis for complexity very well describes that Naive Bayes mine algorithm utilizes optimum time and space complexity than other two algorithms which could be best proposed for accuracy metrics. Comparatively merits are better than the other two algorithms because of increased predictive accuracy rate which may have slight variation with the real time.

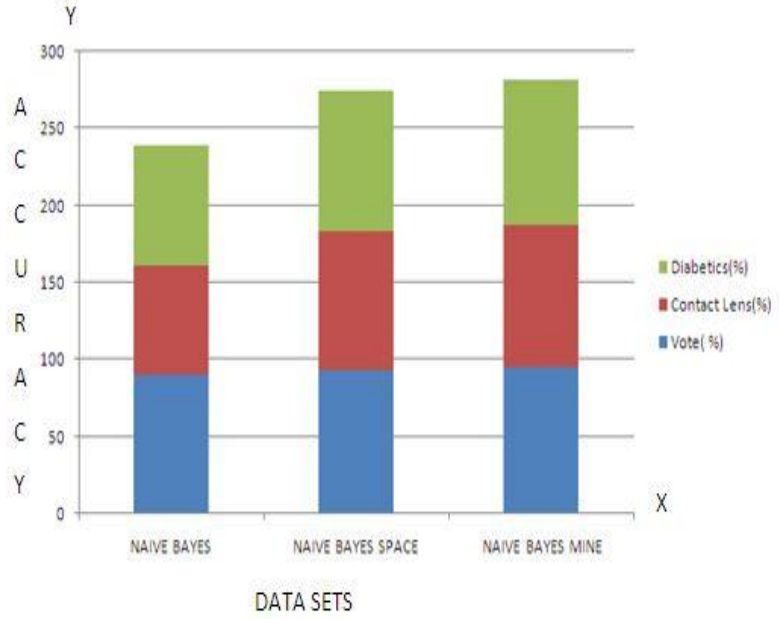

Fig. 3 Performance Accuracy Graph

\section{CONCLUSION}

The dataset of diabetics, contact lens and voting has tested with the existing and proposed algorithm. The proposed algorithm predicted as $30 \%$ higher accuracy rate is achieved in Naïve Bayes Space and Naïve Bayes Mine algorithm when compared to existing Bayesian algorithm. Our Proposed algorithm optimized Space and time Complexity for large data sets.

\section{REFERENCES}

1 Ariana Broumand, Mohammad Shahrokh Esfahani, Byung-Jun Yoon, Edward R. Dougherty "Discrete Optimal Bayesian Classification With Error-Conditioned Sequential Sampling”, Elseiver Pattern Recognition, Vol. 48, No. 32, pp. 3766-3782, March 2015.

2 Candace Berrett, Catherine A. Calder, "Bayesian Spatial Binary Classification", Elseiver Spatial Statistics, Vol. 16, No. 23, pp. 72-102, May 2016.

3 Scott Maclean, George Labahn, "A Bayesian Model For Recognizing Handwritten Mathematical Expressions", Elseiver Pattern Recognition, Vol. 48, No. 34, pp. 24332445, October 2014.

4 Guizhen Zhang, Vinh V. Thai, "Expert Elicitation And Bayesian Network Modeling For Shipping Accidents", Elseiver Safety Science , Vol. 87, No. 22, pp. 53-62, April 2015.

5 Kevin Aagaard, Julie L. Lockwood, Edwin J. Green, “A Bayesian Approach For Characterizing Uncertainty In Declaring A Population Collapse", Elsevier Ecological Modelling, Vol. 328, No. 25, pp.78-84, March 2016.

6 Hamid Reza Tohidypour, Student Member, Mahsa T. Pourazad, And Panos Nasiopoulos, "Probabilistic Approach For Predicting The Size Of Coding Units In The Quad-Tree Structure Of The Quality And Spatial Scalable Hevc", IEEE Transactions On Multimedia, Vol. 18, No. 10, pp.183-195, February 2016.

7 Sebastian Tschiatschek And Franz Pernkopf, "On Bayesian Network Classifiers With Reduced Precision Parameters", IEEE Transactions On Pattern Analysis And Machine Intelligence, Vol. 37,No. 11, pp.774-785, April 2015. 
8 Boyla O. Mainsah, Kenneth D. Morton, Member, Ieee, Leslie M. Collins, Eric W. Sellers, And Chandra S. Throckmorton ,"Moving Away From Error-Related Potentials To Achieve Spelling Correction In P300 Spellers",IEEE Transactions On Neural Systems And Rehabilitation Engineering, Vol. 23,No. 21, pp.737-743, September 2015.

9 Q. Mao ,I. W. Tsang, S. Gao, L. Wang, "Generalized Multiple Kernel Learning With Data-Dependent Priors" ,IEEE Transactions On Neural Networks And Learning Systems, Vol. 26,No. 22, pp.1134-1148, June 2015.

10 Jeevanand Seshadrinath, Bhim Singh, Bijaya Ketan Panigrahi, "Incipient Interturn Fault Diagnosis In Induction Machines Using An Analytic Wavelet-Based Optimized Bayesian Inference",IEEE Transactions On Neural Networks And Learning Systems, Vol. 25, No. 13, pp.990-1002, May 2014.

11 Atizez Hadricha, Mourad Zribia, Afif Masmoudib, "Bayesian Expectation Maximization Algorithm By Using B-Splines Functions: Application In Image Segmentation", Elsevier Journal On Mathematics And Computers In Simulation, Vol. 120, No. 15, pp. 50-63, July 2015.

12 SFuster-Parraa,B, P. Tauler, M. Bennasar-Veny, A. Ligeza, A.A. López-González, A. Aguiló, “ Bayesian Network Modeling: A Case Study Of An Epidemiologic System Analysis Of Cardiovascular Risk", "Elsevier Journal Of Computer Methods And Programs In Biomedicine", Vol.126,No. 23, pp. 128-142, December 2015.

13 Wei Tiana, Song Yanga, Zhanyong Li, Shen Wei, Wei Pand, Yunliang Liua, "Identifying Informative Energy Data In Bayesian Calibration Of Building Energy Models", Elsevier Journal On Energy And Buildings, Vol.14, No. 33, pp. 1-14, March 2016.

14 Javier Galaperoa, et.al,"Identifying Risk Factors For Ovine Respiratory Processes By Using Bayesian Networks", Elsevier Journal On Small Ruminant Research, Vol.136, No. 21, pp.113-120, January 2016. 